\title{
Mitochondrial manipulations
}

\author{
Recent major scientific advances in the ability to replace mitochondria harboring pathological DNA mutations in \\ the germ line could soon be harnessed to tackle mitochondrial diseases. But ethical and scientific concerns require \\ continued debate before such an approach can reach the clinic.
}

nherited mitochondrial diseases are caused by maternally derived mutant mitochondrial DNA (mtDNA). Normal and mutated mtDNA can coexist in the same cell, a phenomenon called heteroplasmy, but once the heteroplasmy passes a certain threshold (typically 60-85\%), disease symptoms often occur. Although such diseases are rare and symptoms are heterogeneous, they can be devastating. Currently, therapeutic remedies are lacking, and affected parents are left with few choices for having healthy children. Such options can include preimplantation genetic diagnosis (PGD) of embryos to reduce-though not eliminate-transmission of mutated mitochondria or prenatal testing, which is often limited to known mutations and is prone to error.

Newly developed approaches, however, offer the potential to tackle this unmet clinical need by eliminating the risk of disease transmission to children and to future generations. In particular, maternal spindle transfer (MST) and pro-nuclear transfer (PNT) allow the ability to transfer nuclear genetic material from an oocyte or zygote, respectively, to an enucleated recipient cell. This would allow a mother with a mitochondria disease to be the genetic parent of an offspring without passing on mutant mtDNA, as the donor healthy egg or zygote would be the source of the mtDNA. Such approaches have been tested using Macaque oocytes, which developed into viable offspring, and in human oocytes and zygotes up to the blastocyst stage.

The UK has spearheaded a movement to establish the appropriate road map to put mitochondrial replacement into clinical practice. For the last three years, the Human Fertilisation and Embryology Authority (HFEA), which regulates reproductive techniques in the UK, has explored the potential use of these approaches. Because MST and PNT alter the germ line, the UK government will have to amend the law that regulates reproductive techniques. To date, although the HFEA has concluded that some scientific caveats still need to be considered, overall they feel there is sufficient evidence of safety and efficacy to warrant use in the clinic for these specific applications. Soon Parliament will debate this issue, encouraged by support from the HFEA expert committee, the public and an influential British bioethics council.
Meanwhile, in the US, where no regulations exist-only guidelines-that oversee reproductive approaches in the clinic, this debate is lagging behind. As the germ line will be manipulated, the US Food and Drug Administration (FDA) has jurisdiction over whether clinical trials can go forward using these techniques. But only recently has it decided to weigh in. In February it held its first workshop to assess the existing evidence to support human clinical trials to prevent mitochondrial disease using MST or PNT. Although ethical issues were part of the discussion, the advisory committee charged by the FDA to explore the use of these approaches was asked to address only the necessary preclinical data, the design of clinical trials, the potential health risks for trial participants and how to mitigate such risks associated with mitochondrial manipulation. The FDA expert panel concluded it was best to hold off on further consideration of conducting such trials until there is more preclinical data addressing existing scientific concerns.

Many of the scientific and clinical matters raised have been contentious and so far seem open ended. First, there is no convincing preclinical evidence to prove the long-term safety of MST and PNT. Second, animal testing using these techniques has yet to be done to show their efficacy in preventing mitochondrial disease in the offspring. Third, mutated mitochondria may be carried over to the healthy cell during the nuclear transfer procedure. This may pose future problems, as the mutant mitochondria may have a replication advantage over the normal mitochondria, contributing to reemergence of heteroplasmy and disease. Because this amplification has been observed in oocytes, this is particularly problematic for female progeny. However, preclinical data indicate that this carryover is low or even undetectable at both the embryonic and somatic cell stage (see references in http://1.usa.gov/1r5iiL0). Finally, the existence of different mitochondrial haplotypes in humans could cause nuclear-mitochondrial incompatibility, which may also affect mitochondria-induced epigenetic modification of the nuclear genome. Published studies, however, do not suggest any serious concerns in this regard, and matching mitochondrial genotypes beforehand could minimize inadvertent damage to the embryo. 


\section{EDITORIAL}

Taking into account the underlying science, what needs to be considered in the clinical setting? The first step would be to identify which women would be eligible for these techniques. Given the disease variability, it will be hard to predict who will benefit. Women with severe disease or specific mutations or those with high heteroplasmy or near homoplasmy (but who are sufficiently healthy to go through pregnancy) may be proper candidates as PGD might not be helpful, given that most of their embryos will most likely be affected. Also, as MST and PNT may cause chromosomal abnormalities, screening of embryos could help prevent potential damage to the baby. Finally, monitoring of fetal development and long-term follow up of the families by looking at potential onset of disease and mitochondrial function and number in the offspring will help evaluate safety and efficacy.

Although expert committees in both countries were asked to assess solely the preclinical data on MST and PNT, ethical and social concerns have also been voiced as mtDNA is inherited, and germ-line alterations will be passed on to future generations. Some of these concerns may derive, in part, from a lack of complete understanding of the biology of mitochondria. The mtDNA only encodes for a small number of proteins involved in energy production, which, although important for health, will probably have no impact on important human traits. Also, neither technique causes overall changes in the genomic DNA or alteration of genes at will, nor can they be considered reproductive cloning. In addition, all stakeholders should understand what these techniques are-and aren't —and that they are only being considered to replace abnormal mitochondria to prevent untreatable diseases.

Advocates and opponents are now raising their voices to either move this potential therapeutic approach forward or put it on hold until further research proves more compelling or ethical issues can be surmounted. The multidisciplinary efforts undertaken in the UK and the willingness of the FDA to explore science-based evidence to allow the use of such techniques in mothers with mutated mitochondria are encouraging. Despite existing gaps in the preclinical knowledge, the perceived low risk posed by these techniques will probably reassure the UK to commence the necessary legal actions to permit the use of mitochondrial replacement in the clinic in the near future. The FDA may revisit this issue in a few years. We hope additional studies will convince the public and regulatory bodies of the benefits of these techniques for those individuals who most need help to avoid transmitting mitochondrial diseases to their children. Although ethical and societal concerns should be properly weighed, we envision that the continued preclinical development of these therapies and the clinical validation of their safety and efficacy will help alleviate concerns and move treatment options forward. 